\title{
$1 \quad$ Adsorbate chemical environment-based machine learning framework for heterogeneous catalysis
}

\author{
* Equal contribution \\ † Corresponding authors \\ Pushkar G. Ghanekar ${ }^{1 *}$, Siddharth Deshpande ${ }^{1 *}$, and Jeffrey Greeley ${ }^{1 \ddagger}$ \\ 1 Davidson School of Chemical Engineering, Purdue University, West Lafayette, \\ Indiana 47907, USA
}

\begin{abstract}
\end{abstract}
Heterogeneous catalytic reactions are influenced by a subtle interplay of atomic-scale factors, ranging from the catalysts' local morphology to the presence of high adsorbate coverages. Describing such phenomena via computational models requires generation and analysis of a large space of surface atomic configurations. To address this challenge, we present the Adsorbate Chemical Environment-based Graph Convolution Neural Network (ACE-GCN), a screening workflow that can account for atomistic configurations comprising diverse adsorbates, binding locations, coordination environments, and substrate morphologies. Using this workflow, we develop catalyst surface models for two illustrative systems: (i) NO adsorbed on a $\mathrm{Pt}_{3} \mathrm{Sn}(111)$ alloy surface, of interest for nitrate electroreduction processes, where high adsorbate coverages combine with the low symmetry of the alloy substrate to produce a large configurational space, and (ii) $\mathrm{OH}^{*}$ adsorbed on a stepped Pt(221) facet, of relevance to the Oxygen Reduction Reaction, wherein the presence of irregular crystal surfaces, high adsorbate coverages, and directionally-dependent adsorbate-adsorbate interactions result in the configurational complexity. In both cases, the ACE-GCN model, having trained on a fraction $(\sim 10 \%)$ of the total DFT-relaxed configurations, successfully ranks the relative stabilities of unrelaxed atomic configurations sampled from a large configurational space. This approach is expected to accelerate development of rigorous descriptions of catalyst surfaces under in-situ conditions.

Tags: Heterogeneous catalysis, density-functional theory, machine-learning, graph networks 


\section{Introduction}

51 insights. understanding of catalyst properties under realistic reaction conditions, as

54 heterogeneous catalytic reactions are sensitive to the atomic-scale complexities arising 55 from adsorbate-adsorbate interactions at high adsorbate coverages, the local 56 morphology of the catalysts, and variations in the catalysts' surface composition induced 57 by adsorption, among other factors. ${ }^{16-22}$ To successfully overcome these difficulties, 58 efficient generation and analysis of atomistic models is critical and requires development 
59 of methods that can efficiently sample the large configurational space of surface atomic

60 configurations for diverse catalyst compositions and surface structures. ${ }^{23,24}$

61 Herein, we present a generalized screening workflow that seeks to address these

62 challenges. The approach involves systematic enumeration of atomic configurations

63 using graph-based representations. ${ }^{23}$ The relevant chemical and geometric properties

64 of the generated motifs are learned and mapped to the target property of choice using

65 a machine learning model based on a graph neural network architecture, ${ }^{25,26}$ which is

66 termed the Adsorbate Chemical Environment-based Graph Convolution Neural Network

67 (ACE-GCN). ACE-GCN serves as a surrogate model for expensive electronic structure

68 optimization routines and efficiently provides estimates for the target properties of

69 catalyst surfaces, thereby facilitating high throughput evaluation of a large space of

70 complex active site models.

71 The proposed workflow can systematically describe a variety of atomistic

72 configurations comprised of diverse adsorbates, binding locations, coordination

73 environments, and catalyst morphologies. This flexibility is demonstrated in the context

74 of two catalytic systems that are relevant to practical electrocatalytic applications and

75 that represent the typical complexities encountered when developing computational

76 models of heterogeneous catalysts. The first case treats high coverage configurations of

77 the adsorbate $\mathrm{NO}^{*}$ on a $\mathrm{Pt}_{3} \mathrm{Sn}(111)$ terrace surface, wherein a vast surface

78 configurational space resulting from both the reduction in the catalyst surface symmetry

79 due to alloying ${ }^{27-30}$ and the strong binding nature of $\mathrm{NO}^{*}$ yields rich catalytic behavior.

80 This chemistry is of interest in electrocatalytic water treatment strategies, and similar

81 complexities arise in chemistries such as Fischer-Tropsch synthesis and water-gas shift. 
8217,31 With our proposed workflow, all high coverage $\mathrm{NO}^{*}$ configurations $(\sim 3400)$ are

83 analyzed by performing only a small fraction of explicit DFT calculations ( 350$)$. In the

84 second case, the challenge of modeling irregular or defected crystal surfaces, together

85 with strong, directionally-dependent adsorbate-adsorbate interactions, is addressed.

86 High coverage configurations of $\mathrm{OH}^{*}$, known to be stabilized through intermolecular

87 hydrogen bonds ( $\mathrm{H}$-bonding), are analyzed on the $\mathrm{Pt}(221)$ stepped and $\mathrm{Pt}(100)$ square

88 surfaces. These types of interactions can strongly impact the energetics of

89 electrocatalytic reactions such as hydrogen evolution, oxygen reduction, and $\mathrm{CO}$

90 electro-oxidation. ${ }^{32-35}$ An approach inspired by transfer learning is employed, wherein

91 explicit DFT calculations of high coverage $\mathrm{OH}^{*}$ configurations on $\mathrm{Pt}(100)$ terraces $(\sim 200)$

92 are combined with selected calculations of $\mathrm{OH}^{*}$ on $\mathrm{Pt}(221)(\sim 400)$. Using the ACE-GCN

93 approach, and subsequently including a modest number of additional high coverage

94 geometries ( 800) for incremental model improvement, a comprehensive set of high

95 coverage $\mathrm{OH}^{*}$ configurations on the $\mathrm{Pt}(221)$ surface $(\sim 11500)$ is explored to identify low

96 energy adsorbate structures. This generalized approach shows how multiple datasets

97 may be used to incorporate information from diverse catalyst morphologies to efficiently

98 describe complex, low symmetry surfaces with vast configurational spaces in the ACE-

99 GCN framework. ${ }^{36-38}$ Finally, we briefly illustrate the utility of these approaches for

100 determining in-situ catalyst structures under realistic reaction conditions by analyzing

101 the state of Pt(221) surface via an ab-initio Pourbaix analysis. 


\section{Results and Discussion}

104

105

106

107 properties, and (ii) adsorbate structures, which may involve multiple adsorbed species

108 and directionally-dependent adsorbate-adsorbate interactions such as hydrogen

109 bonding. Such chemical complexities yield a large phase space of possible atomic

110 configurations, motivating development of a systematic computational framework to

111 screen configurations with less expense than is required by exhaustive first principles

112 analysis.

\section{Workflow and ACE-GCN Framework}

114

115

116 is divided into four parts: (i) systematic enumeration of unique atomic configurations, (ii)

117 (re)training the surrogate model with data of incremental complexity, (iii) accelerated

118 screening using the surrogate model to identify the most relevant configurations

119 amongst possible geometries, and (iv) electronic structure relaxation of selected

120 structures, which can be used for in-depth mechanistic analysis, or to improve the

121 surrogate model.

122 First, adsorbate configurations are generated by enumerating adsorbate binding

123 locations on the catalyst surface using the SurfGraph algorithm. ${ }^{23}$ This algorithm utilizes

124 graph-based representations to identify and create unique surface adsorbate

125 configurations, systematically accelerating the task of generating complex catalytic 
126 model motifs. ${ }^{23,24}$ Next, ACE-GCN is utilized as a surrogate model for screening the

127 generated motifs. The algorithm captures the geometric and chemical properties of a

128 given surface adsorbate's local environment and maps them to a target property of

129 choice. In this work, ACE-GCN is initially trained on a small subset of relaxed adsorbate

130 configurations, and then utilized as a surrogate model to systematically rank the energies

131 of a much larger number of unrelaxed adsorbate configurations. The approach thus

132 provides a framework to efficiently identify a subset of highly promising candidate

133 structures, as generated by SurfGraph, for subsequent electronic structure relaxation,

134 therefore bypassing the computationally expensive step of DFT-optimizing all possible

135 atomistic configurations. After electronic-structure optimization of the most promising

136 structures, the selected candidate configurations are used to further improve the

137 prediction capabilities of the ACE-GCN model by including them in an expanded training

138 pool, as well as to perform an in-depth analysis of the reaction mechanism. Below,

139 additional descriptions of the ACE-GCN framework, as well as two examples of its

140 application are provided. 
(a)

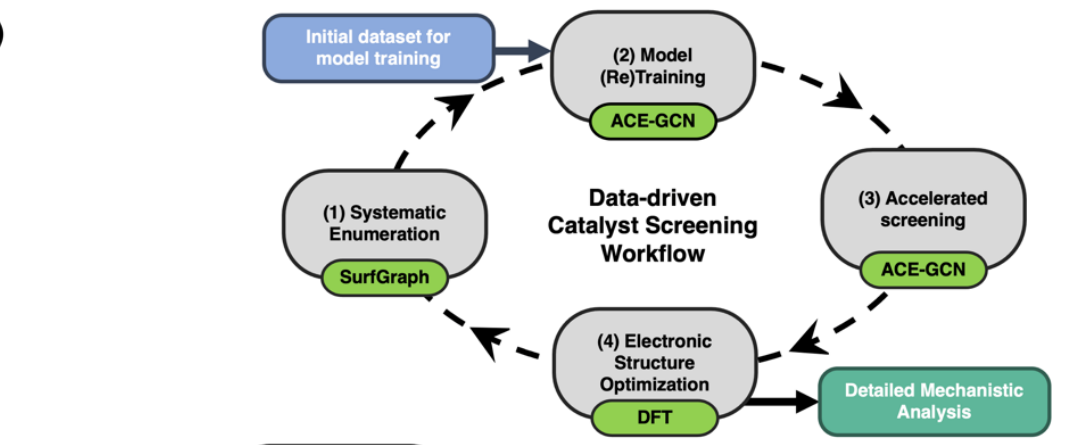

(b)

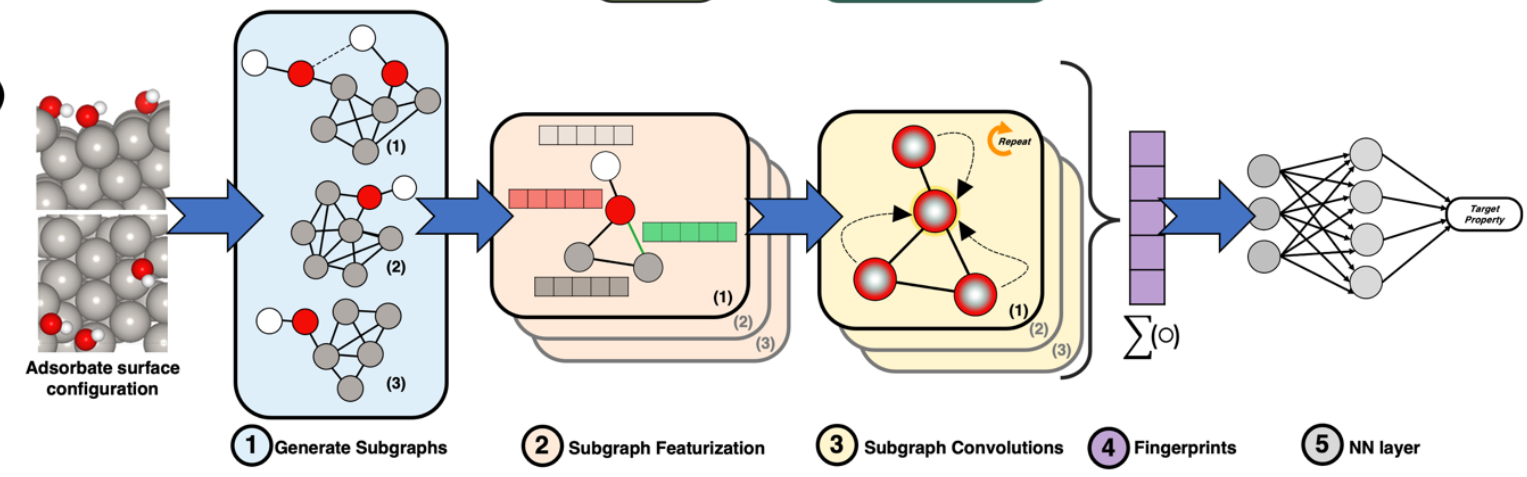

Figure 1: (a) Screening workflow for identifying stable surface adsorbate configurations. The workflow demonstrates an incremental training approach to predict thermodynamically stable catalytic configurations. The cyclic workflow includes the following steps (1) Systematic Enumeration: all possible and unique high coverage surface adsorbate representations are generated using the SurfGraph algorithm, (2) Model Training: ACE-GCN model is (re)trained on selected structures utilizing the relevant surface representations identified in the previous steps. (3) Accelerated Screening: The unrelaxed surface configurations generated in step 1 are ranked using the ACE-GCN model, which is pre-trained on smaller subset of relevant DFT-relaxed cases. (4) Electronic Structure Optimization: selected unrelaxed configurations ranked by ACE-GCN are optimized using electronic structure optimization code of choice and then utilized either for subsequent analysis or to re-train and improve the ACEGCN model.

(b) ACE-GCN algorithm to encode and train high coverage adsorbate configurations. (1) Generate sub-graphs: each configuration is split into multiple subgraphs, as identified by the SurfGraph algorithm. A distinct ego-graph is generated for each adsorbate to encode local geometric and chemical properties around the adsorbate in a subgraph representation. (2) Subgraph Featurization: each atom and its corresponding bond attribute in the subgraph is expressed as a vector representation according to the chemical identity (elemental properties) and spatial bond distance, termed as node and edge features, respectively. (3) Subgraph Convolutions: every node vector in the subgraph is iteratively updated through multiple rounds of graph convolution operations, which account for the atom's geometric and chemical neighborhood using node and edge vectors of the neighboring atoms. (4) Fingerprints: a hierarchical pooling operation condenses all subgraphs for every adsorbate into one fingerprint vector. (5) NN Layer: the fingerprint vector is passed to a feed-forward neural network (NN) which maps it to the target property of choice, such as the average adsorption energy.

142 Adsorbate chemical environment-based graph neural networks

The ACE-GCN framework is based on a graph neural networks (GNN)

144 architecture. ${ }^{25,39}$ Graph-based learning, wherein small molecules or crystals are 
145 presented as undirected graphs with atoms described as nodes and edges representing

146 the connections between the atoms, has been used to accurately account for the

147 underlying structural and chemical properties for a diverse class of materials including

148 small molecules, ${ }^{39}$ periodic materials, ${ }^{25,40}$ metal-organic frameworks, ${ }^{8}$ and selected

149 surfaces. ${ }^{6}$ However, a successful implementation of such graph-based representations,

150 or any surrogate model framework, for complex surface models incorporating a

151 combination of multiple adsorbates, high-coverage ensembles, and complex surface

152 geometries (steps, kinks, and other defects), remains highly challenging. The ACE-GCN

153 model constitutes a simple strategy for treating these sources of complexity.

154 The schematic in Figure 1B shows the steps involved in predicting a target

155 property using ACE-GCN. Each adsorbate surface configuration is initially split into

156 subgraphs (Figure $1 \mathrm{~b}(1)$ ), which are in turn undirected 'ego-graphs' centered around a

157 particular adsorbate generated using the SurfGraph algorithm. These subgraphs

158 explicitly account for the local chemical and structural environment of the adsorbate and

159 can accurately represent the complexities arising from the presence of local co-

160 adsorbates, defect sites, and compositional variations, enabling a systematic

161 description of the surface-adsorbate and adsorbate-adsorbate interactions. Next, every

162 node and edge attribute of the subgraph is expanded as a vector representation of the

163 user-defined chemical and geometric features (Figure $1 \mathrm{~b}(2)$ ). To systematically capture

164 the geometric and chemical environment features surrounding every node, the node

165 feature vector for each node in a subgraph is iteratively updated based on the

166 neighboring environment through multiple rounds of graph convolution (message-

167 passing) steps (Figure $1 \mathrm{~b}(3))$. Next, hierarchical pooling-like operations are performed to 
168 condense multiple arbitrary-sized subgraphs into a fixed-length vector fingerprint (Figure

$169 \mathrm{1b}(4))$. This strategy allows ACE-GCN to successfully operate on cases containing

170 arbitrary numbers of adsorbates and associated neighbors. Finally, the fingerprint vector

171 is used as an input to a fully-connected neural network to predict the property of interest,

172 such as the average adsorption energy (Figure $1 \mathrm{~b}(5)$ ). Additional information regarding

173 the attributes considered for chemical and geometric encoding, the graph convolution

174 equation, supplemental indexing, and hierarchical pooling operations is provided in the

175 Methods section.

177 Modeling complex heterogeneous catalytic systems using the ACE-GCN 178 scheme

180 We consider two representative heterogenous catalytic reactions to illustrate the 181 application of ACE-CGN. First, we analyze the stability of high coverage configurations 182 of $\mathrm{NO}^{*}$ (**) represents an adsorbed moiety) adsorbed on a $\mathrm{Pt}_{3} \mathrm{Sn}(111)$ surface, and

183 second, we determine the most energetically favorable high coverage configurations of $184 \mathrm{OH}^{\star}$ adsorbed on $\mathrm{Pt}(221)$ and $\mathrm{Pt}(100)$ surfaces. Below, we briefly describe the features 185 of the ACE-CGN algorithm that are highlighted in each example, and in subsequent 186 sections, we provide details of the results.

187 The first example demonstrates how the concepts of crystal graph generation and 188 neural network analysis can accelerate analysis of the large configurational spaces 189 arising from the presence of high coverages of adsorbates (in this case, $\mathrm{NO}^{*}$ ) on multi190 elemental alloy surfaces. Both surface and bulk alloying introduce a plethora of surface 191 adsorption sites, thereby decreasing the symmetry of the surface and increasing the 
192 number of distinct adsorption configurations. As shown in Figure 2a, even for a single

$193 \mathrm{NO}^{*}$ adsorbate, twice as many distinct adsorption configurations exist on $\mathrm{Pt}_{3} \mathrm{Sn}(111)$ as

194 on a pure $\mathrm{Pt}(111)$ surface. This configurational space increases exponentially as the

195 coverage of surface adsorbates increases (Figure 2(b)(i)). Considering between 1 and 6

$196 \mathrm{NO}^{*}$ molecules, corresponding to surface coverages between 1/12 and 1/2 ML

197 (monolayers), and neglecting active sites that incorporate 'Sn' atoms, there are

198 approximately 3400 unique adsorbate configurations with 2500 configurations for the 5

199 and $6 \mathrm{NO}^{*}$ cases alone. A recent publication explored this $\mathrm{NO} / \mathrm{Pt}_{3} \mathrm{Sn}(111)$ phase space

200 using an evolutionary algorithm-based scheme, and the present work leverages this prior

201 experience to test and validate the ACE-GCN workflow. ${ }^{17,41}$

(a)

(i) $\mathrm{Pt}_{3} \mathrm{Sn}$ (111)

(ii) Pt (100)

(iii) Pt (221)

(iv) H-bond
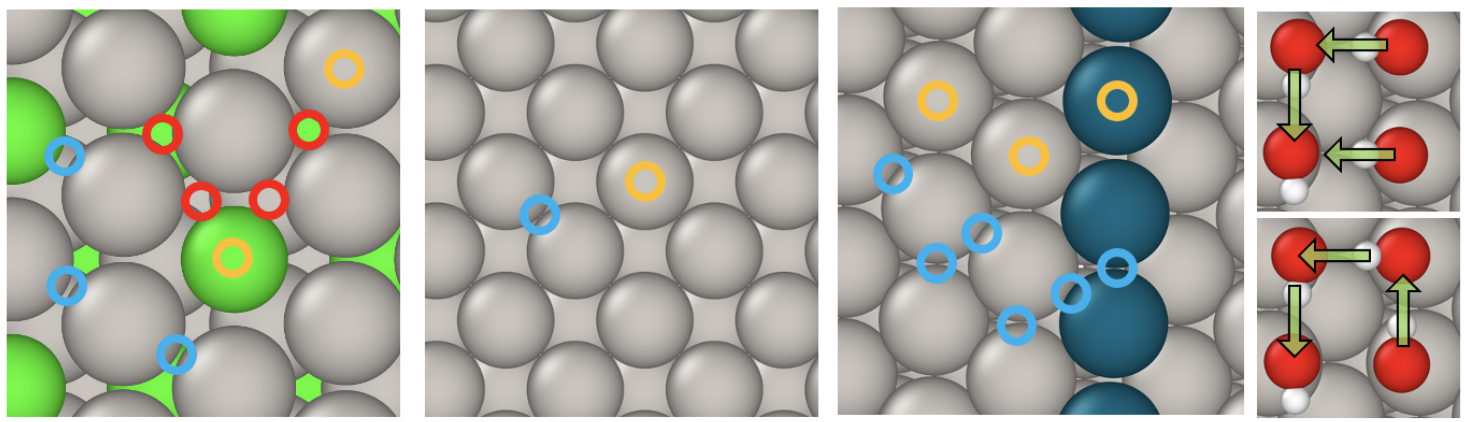

(b)

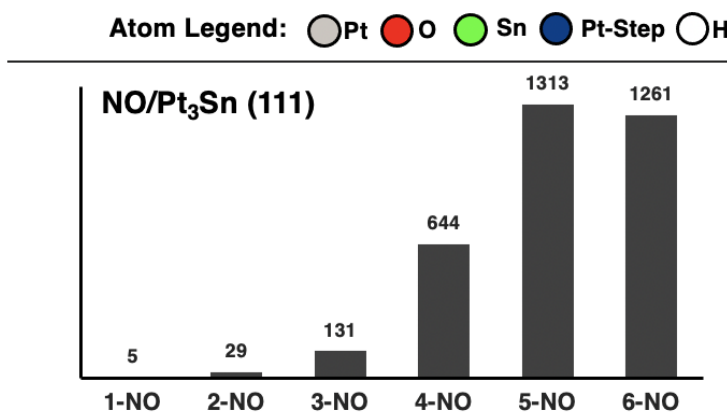

Site Legend: Top Bridge Hollow

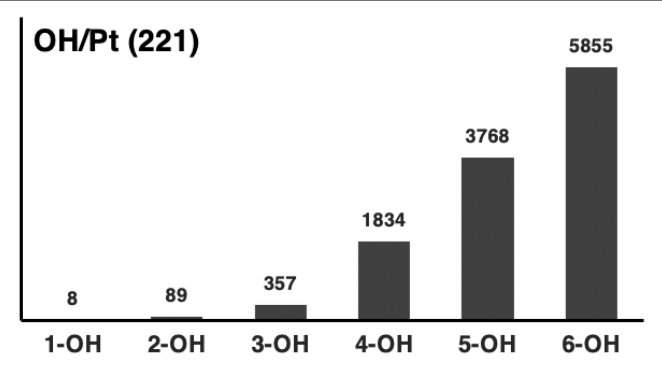

Figure 2: Catalyst configurations analyzed with ACE-CGN. (a) Structural motifs considered in the catalyst models: (i) alloying ( $\left.\mathrm{Pt}_{3} \mathrm{Sn}(111)\right)$, (ii) diversity of binding sites on $\mathrm{Pt}(100)$ and (iii) $\mathrm{Pt}(221)$ (terrace in gray, step in blue) surfaces, and (iv) directionally-dependent intermolecular interactions between adsorbates, such as $\mathrm{OH}^{\star}$. $\mathrm{Green}$ arrows show the direction of $\mathrm{H}$-bonding for each hydroxyl group. (b) The total number of unique surface 
configurations, as a function of adsorbate coverage, for $\mathrm{Pt}_{3} \mathrm{Sn}(111)$ and $\mathrm{Pt}(221)$. All configurations are generated using the SurfGraph algorithm.

204 The second example demonstrates how high coverage configurations of adsorbates

205 may be enumerated on surfaces with defects such as steps and non-hexagonal 206 geometries. This case, which focuses on $\mathrm{OH}^{\star}$, explicitly considers the effect of adsorbate

207 directionality, stemming from intermolecular hydrogen bonding, on the configurational 208 space. Figure 2a(iii) shows a top view of the Pt(221) step surface, which has a three209 atom wide terrace resembling the $\mathrm{Pt}(111)$ surface. The number of possible $\mathrm{OH}^{\star}$ 210 configurations on $\mathrm{Pt}(221)$ is significantly larger than that on terrace models such as

$211 \mathrm{Pt}(100)$ (Figure 2a(ii)) or $\mathrm{Pt}(111)$, since each row of $\mathrm{Pt}$ atoms in $\mathrm{Pt}(221)$ has a unique

212 coordination environment, necessitating separate consideration of adsorption sites on

213 each row of $\mathrm{Pt}$ atoms parallel to the step edge. Additionally, for given $\mathrm{OH}^{*}$ positions on

214 the surface, several hydrogen bonding networks are possible, and since each may have

215 a very different energy, ${ }^{42}$ it is important to explicitly enumerate all such networks (Figure

$2162 \mathrm{a}(\mathrm{iv}))$. Directed graphs, in turn, are an efficient means of incorporating adsorbate

217 directionality into graph-based representations. Initially, all possible O-O pairs that can

218 form hydrogen bonds are determined, following which all unique hydrogen bonded

219 networks amongst the different pairs are estimated (see Methods section for more

220 information). Every hydrogen bond is explicitly encoded as an additional edge attribute

221 in the subgraph generation in ACE-GCN. An illustrative example is presented in Figure

222 2a(iv), wherein two possible $\mathrm{H}$-bonding configurations for $4-\mathrm{OH}^{*}$ on $\mathrm{Pt}(221)$ are shown.

223 Figure $2 b$ (ii), in turn, shows the histogram of the number of configurations as a function

224 of $\mathrm{OH}^{*}$ coverage, which were generated by considering both top and bridge sites till 3 
$225 \mathrm{OH}^{*}$ (coverage of $1 / 4 \mathrm{ML}$ ), and subsequently for the cases of $4,5,6 \mathrm{OH}^{*}(1 / 3,5 / 12$ and

$2261 / 2 \mathrm{ML}$ respectively), only top sites were added. The total configurations are $\sim 12000$,

227 while 1834,3768 , and 5855 configurations are found for the 4,5 , and $6 \mathrm{OH}^{\star}$ cases $(1 / 3$,

$2285 / 12$ and 1/2 ML coverages), respectively. As described further below, we use ACE-

229 GCN to efficiently probe these complex configurational spaces, and we additionally

230 illustrate how the approach can be used to combine insights from diverse datasets, in a

231 strategy reminiscent of transfer learning, ${ }^{36-38}$ by including $\mathrm{OH}^{*}$ adsorption on the

232 geometrically distinct $\mathrm{Pt}(100)$ surface, to yield improved predictions.

233 Estimating most relevant high coverage configurations of $\mathrm{NO}^{*}$ on a $\mathrm{Pt}_{3} \mathrm{Sn}(111)$ alloy 234 catalyst

235 As shown in Figure $2 b(i)$, the total number of unique initial configurations for 1-6

$236 \mathrm{NO}^{*}$ adsorbed on a $\sqrt{12} \times \sqrt{12} \mathrm{Pt}_{3} \mathrm{Sn}(111)$ unit cell (coverage range of $1 / 12-1 / 2 \mathrm{ML}$ )

237 are on the order of $\sim 3400$, with roughly 2500 configurations for the 5 and $6 \mathrm{NO}^{*}$ cases

238 (5/12 and $1 / 2 \mathrm{ML}$ ) alone. The goal of the proposed screening strategy (Figure 1), utilizing

239 ACE-GCN, is to systematically develop a surrogate model, which describes the

240 important interactions governing the stability of low coverage $\mathrm{NO}^{*}$ models $\left(1 / 2 / 3 / 4 \mathrm{NO}^{*}\right.$

241 or $1 / 12-4 / 12 \mathrm{ML}$ coverage), and to use the resulting insights to efficiently screen the

242 vast number of high coverage configurations ( $5 / 6 \mathrm{NO}^{*}, 5 / 12$ and $1 / 2 \mathrm{ML}$ coverage) with

243 minimal additional computational effort. First, an ACE-GCN model is trained on the

244 average $\mathrm{NO}^{*}$ binding energies of all of the low coverage $\left(1,2\right.$, and $3 \mathrm{NO}^{*}, 1 / 12$ to $\left.1 / 4 \mathrm{ML}\right)$

245 DFT-relaxed structures (see the detail for the 1-3 NO* model fit in the Supplemental

246 Information S4), and next, the model is used to predict binding energetics for the 4-NO*

247 (1/3 ML) case. Based on these ACE-GCN predictions, 100 energetically stable and 100 
248 unstable candidates (200 total) of the 644 possible $4-\mathrm{NO}^{*}$ configurations, are then

249 selected. These configurations are relaxed using DFT and added to the incremental

250 model training. Figure $3(a-b)$ shows the parity plots for the training and validation sets

251 for the new $1 / 2 / 3 / 4-\mathrm{NO}^{*}$ dataset (1/12 to $1 / 3 \mathrm{ML}$ coverages). The model fits the target

252 property, average $\mathrm{NO}^{*}$ binding energy, with a mean absolute error of $0.02 \mathrm{eV}$ for training

253 and validation sets, demonstrating that the ACE-GCN architecture can distinguish

254 amongst different coverages through representations consisting of subgraph-based

255 graph convolutions and hierarchical pooling. Next, the modified ACE-GCN model,

256 trained on the exhaustive $1 / 2 / 3-\mathrm{NO}^{*}$ ensemble and some $4-\mathrm{NO}^{*}$ data points, is used to

257 rank the unrelaxed $5-\mathrm{NO}^{*}$ and $6-\mathrm{NO}^{*}$ configurations (5/12 and $1 / 2 \mathrm{ML}$ coverages),

258 generated through SurfGraph, as shown in Figure 3 (c). This dataset is comprised of

2591314 and 1261 configurations for 5 and $6 \mathrm{NO}^{*}$, respectively. In the figure, the $\mathrm{x}$-axis

260 represents the ACE-GCN predicted average binding energy of the initial unrelaxed 5/6-

$261 \mathrm{NO}^{*}$ configurations, and the y-axis gives the corresponding DFT relaxed energy (for

262 clarity, only those $\mathrm{NO}^{*}$ configurations whose binding locations did not change post-DFT

263 relaxation are plotted; additional discussion is provided in the Supplemental Information

264 S4). Importantly, the top $10 \%$ lowest energy unrelaxed configurations identified by ACE-

265 GCN include the most stable DFT relaxed atomistic configurations for both the 5 and 6

$266 \mathrm{NO}^{*}$ cases, and, no additional stable configurations were found after DFT relaxation that

267 were not already identified by SurfGraph (see Supplemental Information for additional

268 details). These results, taken together, strongly suggest that the combination of

269 SurfGraph and ACE-GCN is capable of efficiently identifying all stable high coverage

270 configurations for $\mathrm{NO}^{*}$ adsorption. 
Additionally, the ACE-GCN model captures important information regarding the

272 governing interactions dictating the adsorption geometries of $\mathrm{NO}^{\star}$ on $\mathrm{Pt}_{3} \mathrm{Sn}(111)$. From

273 our recent analysis, ${ }^{23}$ it is known that higher coverages of $\mathrm{NO}^{*}$ are stable in mixed top

274 and bridge configurations on this surface, while combinations of bridge and threefold

275 sites are unstable. The ACE-GCN model captures this insight, without any explicit user

276 input, using only the low coverage (1/2/3/4 NO*, 1/12 to $1 / 3 \mathrm{ML})$ data, and, as described

277 above, efficiently identifies the energetically most stable 5-NO* and 6-NO* (5/12 and 1/2

$278 \mathrm{ML}$ ) configurations. The low energy configurations, in turn (shown in their final

279 configurations post-DFT relaxation in region (i) and (ii) in Figure 3(c)), consist of NO

280 occupying the top and bridge sites on $\mathrm{Pt}_{3} \mathrm{Sn}$. In contrast, higher energy configurations,

281 also shown in region (ii) in Figure 3(c), consist of $\mathrm{NO}^{*}$ occupying a mixture of bridge and

282 hollow sites, and are also accurately identified by the ACE-GCN surrogate model. Finally,

283 it is interesting to note that the degree of restructuring of the adsorbate site after DFT

284 relaxation is directly correlated with the stability of a given configuration as predicted

285 using ACE-GCN. The sites predicted to be the most unstable by ACE-GCN underwent

286 the largest change in the adsorbate position after relaxation. Additional details on the

287 model's prediction capabilities as a function of different training data sets, and further

288 discussion on reconstructed $\mathrm{NO}^{*}$ configurations, are included in Supplemental 289 Information S4. 
(a)

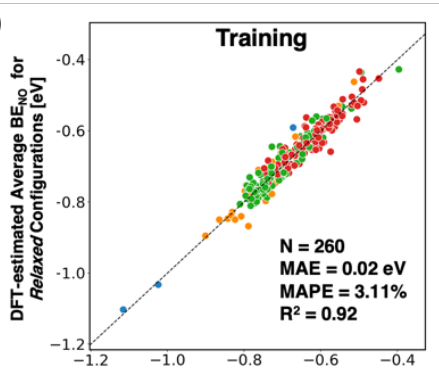

(b)

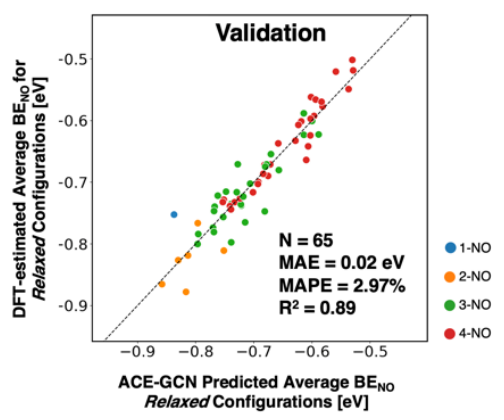

(c)
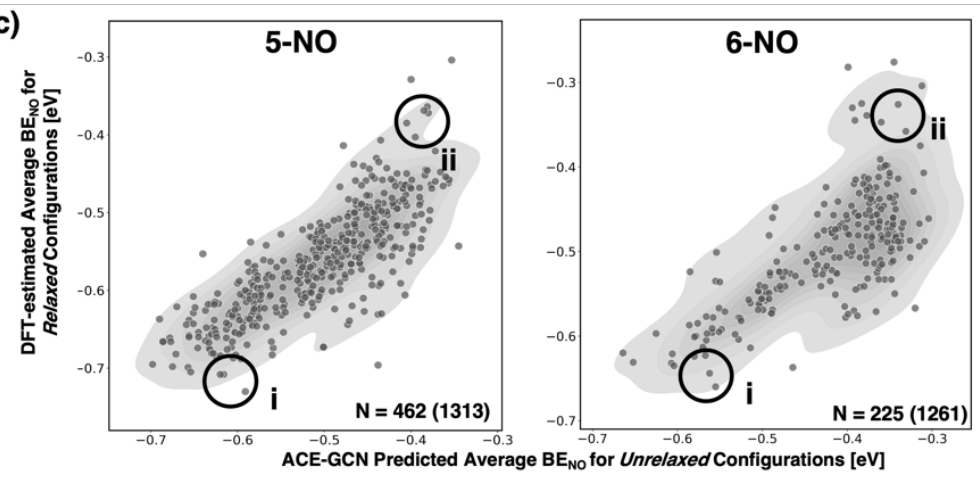

i.

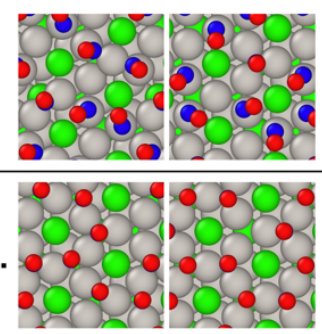

i.

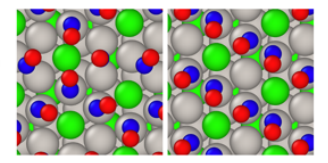

ii.

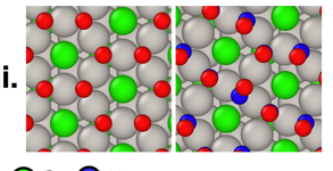

Figure 3: Configurational analysis of $\mathrm{NO}^{*}$ adsorption on $\mathrm{Pt}_{3} \mathrm{Sn}(111)$, where $\mathrm{ACE}-\mathrm{GCN}$ is used to predict energetics of the unrelaxed configurations generated using SurfGraph. (a) and (b) correspond to training and validation parity plots for an ACE-GCN model with NO* configurations consisting of 1-4 NO molecules. (c) gives predictions of the ACE-GCN model, trained on configurations of 1-4 $\mathrm{NO}^{*}$ molecules, for stability of unrelaxed 5 and 6 NO configurations generated with SurfGraph. The predicted average BE of the unrelaxed configurations is plotted on the $x$-axis, while the final energy of the same configurations after DFT relaxation is plotted on the $y$-axis. Only configurations where the binding location of the NO* did not change after DFT relaxation are included. The ACEGCN algorithm successfully predicts the trends in adsorption energies based solely on the unrelaxed configurations generated by SurfGraph. Selected relaxed low and high energy configurations are shown in insets (i) and (ii), respectively.

These results strongly suggest that, through selective incorporation of a small

291 subset of data points of increasingly higher coverages, the ACE-GCN model, trained

292 only on low coverage configurations (1-4 NO*, 1/12-1/3 ML), successfully identifies

293 stable high coverage configurations (5/12-1/2 $\mathrm{ML})$ based solely on the unrelaxed

294 geometries generated from SurfGraph. In comparison to the evolutionary algorithm (EA)

295 scheme used in our previous work, the ACE-GCN model (i) required fewer DFT

296 calculations, 350 versus over 500 data points, compared to the EA, ${ }^{23}$ and (ii)

297 independently captured the underlying chemical and geometric intuition affecting the

298 adsorption energetics. This is an important advantage that becomes even more 
299 significant for larger chemical spaces, where careful analysis of individual configurations 300 and development of chemical intuition could become infeasible.

302 Identifying stable high coverage configuration of interacting hydroxyl adsorbates 303 on defected Pt surfaces

$304 \quad$ This case study illustrates the application of our proposed workflow to adsorbates

305 with directionally-dependent hydrogen bonding on non-hexagonally close-packed

306 single crystal surfaces, $\mathrm{Pt}(100)$ and $\mathrm{Pt}(221)$. The former is chosen as the simplest

307 possible non-hexagonal surface, while the latter represents model step defects that have

308 been shown to exert a significant influence on electrochemical oxygen reduction rates

309 on polycrystalline $\mathrm{Pt}$ electrocatalysts. ${ }^{33,43}$ Along with the comprehensive

310 training/testing/extrapolation strategy for the $\mathrm{Pt}(100)$ and $\mathrm{Pt}(221)$ surfaces, similar to that

311 described for the $\mathrm{NO} / \mathrm{Pt}_{3} \mathrm{Sn}(111)$ case study, we additionally explore the ability of the

312 ACE-GCN framework to synergistically combine insights from training datasets from

313 these two surface morphologies (the benefit of considering such a mixed training dataset

314 on model prediction is further discussed in the Supplemental Information S5). Such

315 strategies will ultimately be key to understanding adsorption configurations on highly

316 complex catalysts, such as polycrystalline nanoparticles, which encompass a variety of

317 different catalyst morphologies. ${ }^{44,45}$

318 The overall workflow is summarized here and described in more detail in

319 subsequent paragraphs. First, a comprehensive training dataset, consisting of

320 configurations with between 1 and $5 \mathrm{OH}^{*}$ molecules per $8 \mathrm{Pt}$ atoms on the $\mathrm{Pt}(100)$

321 surface (coverages of between $1 / 8$ and $5 / 8 \mathrm{ML}$ ), is generated, while a second training

322 set of between 1 and $3 \mathrm{OH}^{*}$ adsorbed per $12 \mathrm{Pt}$ atoms on $\mathrm{Pt}(221)$ (coverages of 1/12 to 
324 much lower than those analyzed on $\mathrm{Pt}(100)$, the total number of training data points are

325 very similar in each case. These datasets, through ACE-GCN, are then combined to

326 efficiently identify low energy adsorption configurations of $\mathrm{OH}^{*}$ on $\mathrm{Pt}(221)$ at much higher

327 coverages $\left(4-6 \mathrm{OH}^{\star} / 12 \mathrm{Pt}\right.$, coverages of $\left.1 / 3-1 / 2 \mathrm{ML}\right)$, where the total number of

328 configurations is exponentially larger (Figure 2c) than the number of configurations

329 associated with similar coverages on $\mathrm{Pt}(100)$.

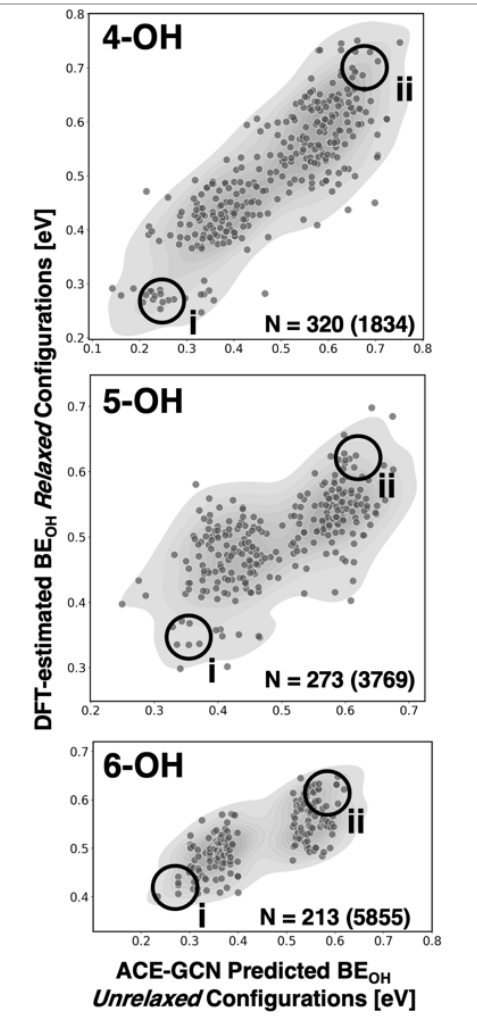

\section{(a) $4-\mathrm{OH}$}

i.

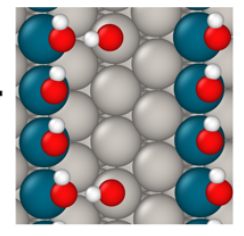

\section{(b) 5-OH}

i.

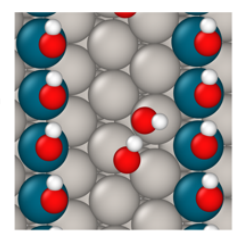

(c) 6-OH

i.

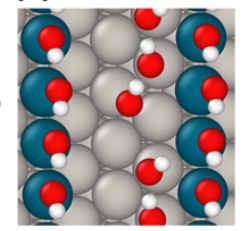

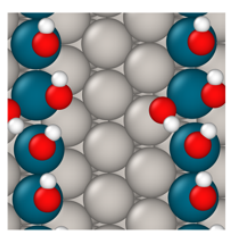

ii.
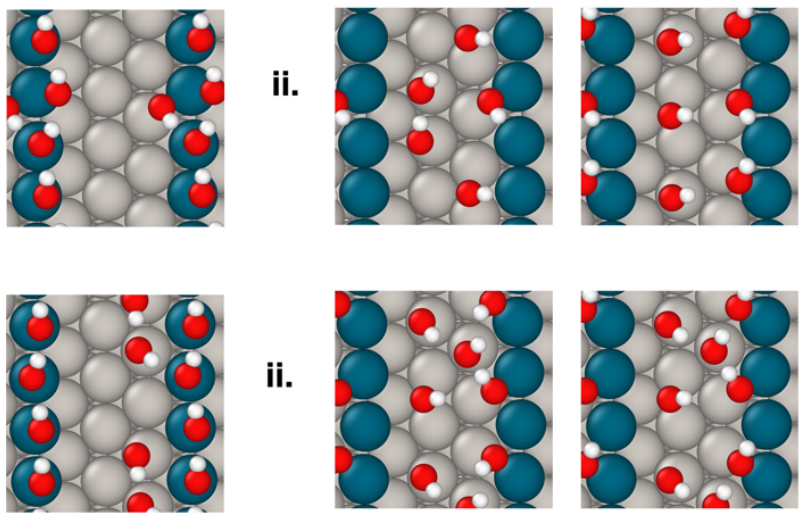

ii.
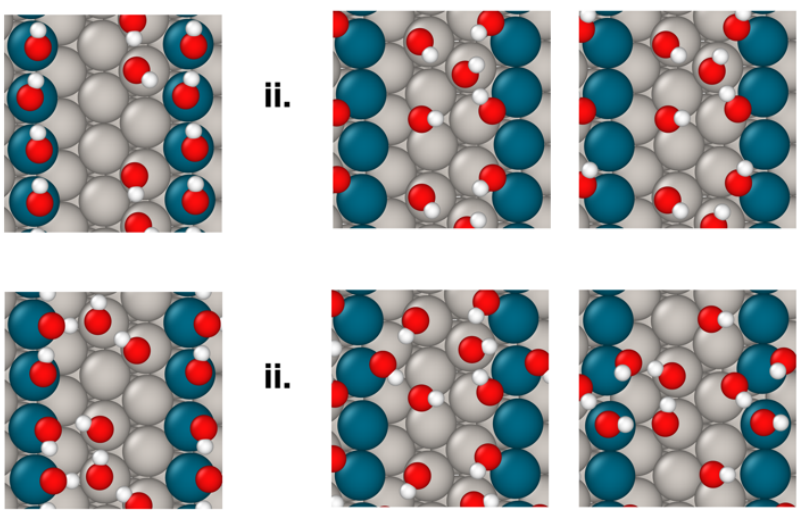

ii.
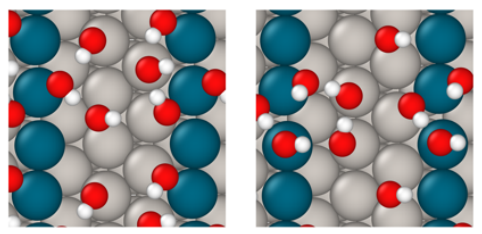

Representative configurations for high coverage $\mathrm{OH}^{\star}$ on $\mathrm{Pt}(221)$

Atom Legend: $\bigcirc$ pt $\bigcirc$ О $\bigcirc$ н Pt-step

Figure 4: Screening high coverage $\mathrm{OH}^{*}$ configurations on $\mathrm{Pt}(221)$. Scatter plots showing average $\mathrm{OH}^{*}$ binding energies of unrelaxed configurations, as predicted by ACE-GCN (x-axis), with DFT-relaxed energies of the corresponding structures (y-axis). A few relaxed configurations showing $\mathrm{OH}^{*}$ species dissociation after DFT relaxation were not included in the plots or model retraining (analysis of dissociated configurations is discussed in the Supplemental Information S5). Numbers in the inset show the total DFT relaxed configurations compared to the total possible structures enumerated by SurfGraph. The ACE-GCN model for each succeeding coverage $(4 / 5 / 6$ $\mathrm{OH}^{*}, 1 / 3$ to $1 / 2 \mathrm{ML}$ ) is trained on configurations with lower coverages (see text for details). 
The $\mathrm{OH}^{*}$ configurations are generated using a modified SurfGraph code that

333 accounts for directional hydrogen bonds among different $\mathrm{OH}^{\star}$ species (see Figure 2(a)

334 for an example). As mentioned above, the ACE-GCN model is initially trained on the

335 dataset comprising of configurations between 1-3 $\mathrm{OH}^{*}$ adsorbates on $\mathrm{Pt}(221)$ and 1-5

$336 \mathrm{OH}^{*}$ on $\mathrm{Pt}(100)$. Next, the ACE-GCN model is used to rank the unrelaxed $4 \mathrm{OH}^{\star} / \mathrm{Pt}(221)$

337 configurations (1834 in total) (1/3 ML coverage), from which 400 configurations,

338 representing a range of energy values and adsorbate binding configurations, are chosen

339 for full DFT relaxation. Figure 4 (top) shows a comparison of the ACE-GCN predicted

340 average binding energies of the unrelaxed $4-\mathrm{OH}^{*}$ configurations and the corresponding

341 DFT relaxed energies. There is a robust correlation between these two quantities,

342 demonstrating that configurations predicted to be low (or high) in energy based on the

343 ACE-GCN predictions of initial unrelaxed geometries track well with post-DFT relaxation

344 results. Shown on the left of the scatter plot are some of the key $4-\mathrm{OH}^{*}$ configurations

345 post DFT-relaxation belonging to the low/high energy $4-\mathrm{OH}^{\star}$ arrangements. The most

346 stable structures, represented by region (i) in the plot, have the Pt-step edge (marked in

347 dark blue) completely occupied, and any additional $\mathrm{OH}^{*}$ moieties have clustered around

348 the Pt-edge to increase the level of hydrogen bonding. In contrast, the high energy

349 structures, as shown in region (ii), are comprised of separated $\mathrm{OH}^{*}$ species, most of

350 which are not directly adsorbed on the Pt step edge, and with relatively few hydrogen

351 bonds. These results indicate that the ACE-GCN model, trained on the diverse data from

$352 \mathrm{Pt}(100)$ and $\mathrm{Pt}(221)$, accurately learns the underlying features that stabilize the $4-\mathrm{OH}^{*}$ on

353 Pt steps. 
Following the scheme laid out in Figure $1(\mathrm{~A})$, higher coverage $\left(5-\mathrm{OH}^{*}, 5 / 12 \mathrm{ML}\right.$ coverage) configurations are generated by using Surfgraph to systematically add an

356 additional $\mathrm{OH}^{*}$ moiety to the exhaustive set of unrelaxed $4-\mathrm{OH}^{*}$ configurations. These

357 configurations are then ranked using a retrained ACE-GCN model incorporating the

358 previously DFT-relaxed $4-\mathrm{OH}^{*}$ configurations in the training set. A few of the identified

359 configurations resulted in dissociated $\mathrm{OH}^{*}$ species after relaxation, and these cases have

360 not been included in the analysis or model retraining (see Supplemental Information S5).

361 Analogous to the $4-\mathrm{OH}^{\star}$ case, a total of 400 unrelaxed configurations, 200 each chosen

362 from high and low energy zones as identified by the ACE-GCN predictions, are selected

363 for DFT relaxation. Finally, a similar strategy is applied when searching for $6-\mathrm{OH}^{\star}$

364 configurations (1/2 ML of coverage), where the emphasis is again placed on high and

365 low energy structures. 3769 and 5855 possible $\mathrm{OH}^{\star}$ configurations exist for the 5 and 6

$366 \mathrm{OH}^{*}$ cases, respectively, of which only about 400 configurations each for 5 and $6 \mathrm{OH}^{*}$

367 cases are evaluated using DFT and about 273 and 213 cases remain undissociated post

368 DFT relaxation. The correlation between the stability of structures predicted via ACE-

369 GCN and those after DFT optimization is again quite reasonable (Figure 4); the quasi-

370 bimodal nature of the 5 and $6-\mathrm{OH}^{*}$ plots is simply the result of our choice to sample high

371 and low energy structures, as predicted by ACE-GCN, for DFT optimization. Further, in

372 line with the chemical intuition developed with lower coverages, for both 5 and $6 \mathrm{OH}^{\star}$

373 cases (5/12 and $1 / 2 \mathrm{ML}$ coverages), the most stable configurations are comprised of

374 clustered $\mathrm{OH}^{*}$ species on the Pt-step edge, whereas unstable cases involve spatially

375 separated $\mathrm{OH}^{*}$ with few $\mathrm{OH}^{*}$ moieties adsorbed on the step edge. We note, however,

376 that despite the reasonable energetic and chemically intuitive predictions from the ACE- 
377 GCN analysis, there can be non-trivial relaxations of the unrelaxed structures, especially

378 for the high coverage cases of 5 and $6 \mathrm{OH}^{*}$ on the surface $(5 / 12$ and $1 / 2 \mathrm{ML})$. We attribute

379 these relaxations to the observation that multiple highly clustered $\mathrm{OH}^{*}$ representations

380 may have similar average $\mathrm{OH}^{\star}$ interaction energies but, may undergo substantially

381 different relaxation during DFT optimization.

382 The $\mathrm{Pt}(221)$ and $\mathrm{Pt}(100)$ analyses demonstrate the capability of ACE-GCN to (i)

383 learn important underlying interactions governing the stability of adsorbates with

384 directionally-dependent interactions, such as $\mathrm{OH}^{*}$, on irregular catalyst models by

385 simulating only about $5-6 \%$ of the total number of possible configurations, and (ii)

386 combine data having different catalytic morphologies, in a transfer learning-inspired

387 approach, to train surrogate models with high efficiency. Such an analysis can aid in

388 developing chemical intuition regarding the underlying interactions that are crucial for

389 stabilizing the adsorbates and understanding the state of the system in realistic reaction

390 environments.

392 Mechanistic implications of high $\mathrm{OH}^{\star}$ coverages for electrochemical 393 reactions on $\mathbf{P t}$

$394 \quad$ Based on the identified $\mathrm{OH}^{*}$ configurations on the irregular Pt surfaces, a detailed

395 thermodynamic and mechanistic analysis to investigate the state of the catalyst surface

396 under electrochemical reaction conditions, such as those relevant to oxygen reduction

397 reaction (ORR), can now be undertaken. Previous reports have demonstrated that (111)

398 terraces on Pt catalysts are among the most active facets for ORR, and recent

399 investigations on irregular crystal facets of $\mathrm{Pt}$, having variable step sizes ((221), (331) and 
400 (211)), suggest high ORR activity on these surfaces, as well. ${ }^{33,43,46} \mathrm{~A}$ mechanistic analysis

401 incorporating the effects of catalyst morphology and $\mathrm{OH}^{*}$ coverages is, in turn, needed

402 to understand these experimentally observed trends. However, the large phase space of

403 possible atomic configurations, especially for the case of stepped catalyst surfaces,

404 makes the analysis challenging.
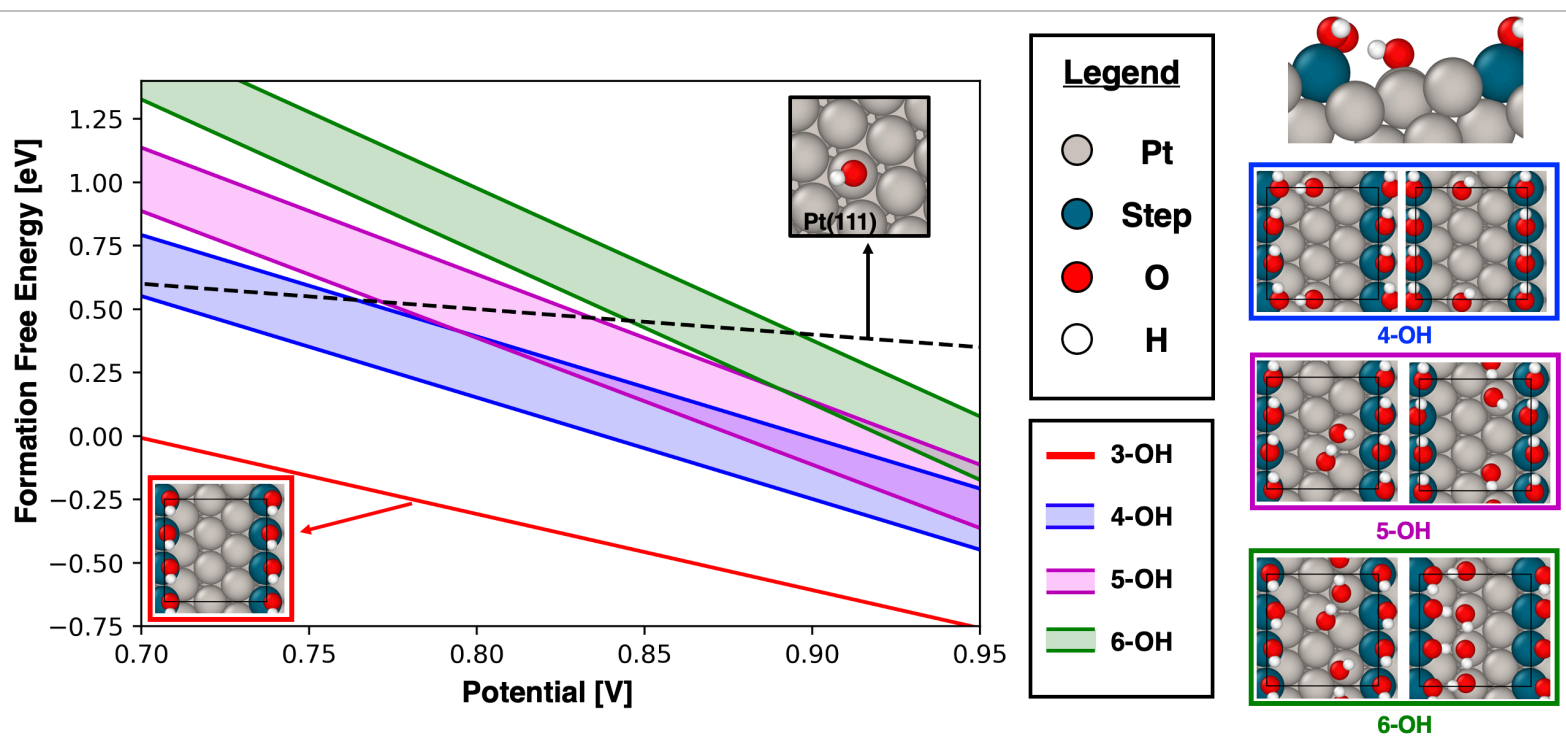

Figure 5: Ab-initio Pourbaix diagram based on binding energies of various $\mathrm{OH}^{\star}$ configurations on $\mathrm{Pt}(111)$ and Pt(211) surfaces. In the potential region of interest for ORR, 0.8-0.9 V, competition between ensembles having 4, 5, and $6 \mathrm{OH}^{*}$ adsorbates on $\mathrm{Pt}(221)\left(1 / 4\right.$ to $1 / 2 \mathrm{ML} \mathrm{OH}^{*}$ coverage) is predicted, and is shown by the overlapping free energy bands. On the rightmost side of the figure, a side view of the most stable $4-\mathrm{OH}^{*}$ configuration in our analysis is shown (the corresponding top view is in the $4-\mathrm{OH}$ inset on the left side). It is observed that the $\mathrm{OH}^{*}$ present on the terrace site immediately below the Pt step edge (termed "back-terrace" in our discussion) has a favorable $\mathrm{H}$-bond with the $\mathrm{OH}^{*}$ absorbed on the edge. Such an arrangement of $\mathrm{OH}^{*}$ moieties is possible due to the particular geometry of the step and edge sites. This arrangement results in appreciable stabilization compared to the scenario where no such $\mathrm{H}$-bond exists (shown in the 4-OH inset, right side). Representative surface configurations for 5 and $6-\mathrm{OH}^{*}$ are also indicated on the insets to the right of the figure, with the most stable configurations on the left of the insets.

407 Pourbaix diagram is generated (Figure 5) to explain the state of the Pt(221) surface under

408 ORR-relevant conditions. For simulations reported in Figure 5, larger unit cells along with

409 higher energy cutoffs and k-points are utilized, with additional details reported in the

410 methods section. The formation free energies of the identified high coverage structures 
411 (4-6 $\mathrm{OH}^{*}$ on $\left.\mathrm{Pt}(221)\right)$ are plotted as a function of the applied external potential vs. the

412 Standard Hydrogen Electrode (SHE). The formation free energy for each $\mathrm{OH}^{\star}$ coverage

413 is presented as an energy band, which is $0.25 \mathrm{eV}$ wide, starting from the energy of the

414 most stable configuration identified using the workflow shown in Figure 1. The

415 schematics on the right side of the Pourbaix diagram show the most stable and selected

416 metastable ( $0.25 \mathrm{eV}$ higher in energy) configurations. In addition, the free energy of

417 the most stable $3 \mathrm{OH}^{*}$ configuration on the $\mathrm{Pt}(221)$ facet, together with that of a single

$418 \mathrm{OH}^{*}$ moiety on $\mathrm{Pt}(111)$, is plotted for reference. The $3 \mathrm{OH}^{*}$ ensemble on $\mathrm{Pt}(221)$, where

419 the $\mathrm{OH}^{*}$ species occupy the Pt-step edge, is identified as the most stable $\mathrm{OH}^{*}$

420 configuration. This result suggests that the Pt edge might be completely poisoned at

421 ORR-relevant conditions (red inset and line in Figure 5). Additional population of $\mathrm{OH}^{*}$ on

422 the surface of the catalyst $\left(4,5\right.$, and $\left.6 \mathrm{OH}^{*}\right)$ shows competition amongst different

423 configurations, especially above applied potentials of $0.8 \mathrm{~V}$ vs. SHE. An interesting

424 feature of the identified high coverage configurations on $\mathrm{Pt}(221)$ is the presence of the

$425 \mathrm{OH}^{*}$ adsorbed on the terrace sites that lie adjacent to and below the Pt step edge. Such

426 a binding configuration is a result of the unique spatial arrangement of $\mathrm{Pt}(221)$ step sites

427 (a representative configuration is shown in Figure 5, right side, top inset). Discovering

428 such a unique $\mathrm{OH}^{*}$ binding arrangement, which, to the best of our knowledge, has not

429 yet been reported elsewhere, speaks to the value that data-driven screening workflows

430 such as ACE-GCN can add in helping to identify interesting regions in the chemical

431 phase space, which can then be further explored rigorously to better understand the 432 complex reaction systems. 
434 possess comparable energies. The most stable $\mathrm{OH}^{*}$ arrangements often exhibit

435 hydrogen bonding between the $\mathrm{OH}^{*}$ moiety on the lower terrace with the $\mathrm{OH}^{\star}$ adsorbed

436 on the Pt edge (Figure 5, inset for $4 \mathrm{OH}^{*}$ case), or they possess a combination of $\mathrm{OH}^{*}$

437 adsorbed on both bridge and top sites in chain-like structures near the step on the upper

438 terrace (Figure 5, inset for 5 and $6 \mathrm{OH}^{\star}$ ).

439 It is important to note that, while the identified structural motifs for high coverage

440 adsorbed $\mathrm{OH}^{*}$ may be relevant to practical ORR catalysis, these configurations only

441 consider stabilization due to adsorbate-adsorbate and adsorbate-substrate interactions

442 and do not explicitly account for interactions between adsorbed hydroxyl groups and

443 ambient water solvent molecules, which can have energies on the order of $0.5-0.6 \mathrm{eV}$

444 per $\mathrm{OH}^{\star} .{ }^{42,47,48}$ To illustrate the effect of such corrections, a black dashed line,

445 representing the $\mathrm{OH}^{*}$ adsorption energy on $\mathrm{Pt}(111)$, is plotted in Figure 5. At an applied

446 potential of $0.8 \mathrm{~V}$ vs SHE, the formation free energy for $1 \mathrm{OH}^{*}$ adsorbed on a top site of

$447 \mathrm{Pt}(111)$ is $0.55 \mathrm{eV}$, excluding any solvent corrections, which is consistent with previous

448 reports. It is only the solvent stabilization that reduces the energy of $\mathrm{OH}^{\star}$ to near zero on

$449 \mathrm{Pt}(111)$ (at $0.8 \mathrm{~V}$ vs. SHE) and hence promotes its reactivity. Since the energy of $1-\mathrm{OH}^{*}$

450 on $\mathrm{Pt}(111)$, devoid of any solvent correction, is comparable to the uncorrected energy of

451 the $4 / 5 / 6-\mathrm{OH}^{\star}$ ensembles on $\mathrm{Pt}(221)$, one might expect that some of these ensembles

452 on Pt(221) would be stabilized under ORR condition and contribute to the ORR activity.

453 Further, it is possible that the solvation correction for the high coverage $4 / 5 / 6-\mathrm{OH}^{*}$ cases

454 (1/4 to $1 / 2 \mathrm{ML}$ of $\left.\mathrm{OH}^{\star}\right)$ on $\mathrm{Pt}(221)$ could be different compared to the correction for the

455 low coverage $\mathrm{OH}^{*}$ ensembles on $\mathrm{Pt}(111)$. To fully capture the impact of solvent- 
456 adsorbate interactions on ORR chemistry, further analysis, rigorously incorporating

457 explicit solvent molecules $\left(\mathrm{H}_{2} \mathrm{O}\right)$, along with ab-initio molecular dynamics analysis to 458 understand the electrode-electrolyte double-layer structure, would be necessary. The 459 identified 4/5/6 $\mathrm{OH}^{\star}$ high coverage configurations provide a strong foundation for 460 undertaking such an analysis, and it is likely that many of the key qualitative conclusions 461 from the analysis, such as the favorable adsorption of $\mathrm{OH}^{*}$ on the step edges and the 462 preference for $\mathrm{OH}^{\star}$ on the lower terrace to interact with the step-adsorbed $\mathrm{OH}^{\star}$ groups, 463 will not be altered by the presence of additional water molecules. 


\section{Conclusions and Outlook}

We present a machine learning-based hierarchical screening workflow to

467 systematically estimate active site morphology for complex heterogeneous surface

468 catalytic reactions. The proposed workflow utilizes the graph theory-based SurfGraph

469 algorithm for systematic enumeration and generation of surface adsorbate

470 representations with variable coverages. The generated models are screened using

471 Adsorbate Chemical Environment-based Graph Convolution Neural Network (ACE-

472 GCN), a graph neural network-based framework, which utilizes the chemical and

473 structural environment of a given adsorbate as the input and maps these features to the

474 target property of choice. Using this workflow, we demonstrate the identification of

475 relevant active site models for heterogeneous catalytic systems relevant to strong

476 binding adsorbates on low symmetry alloyed surfaces and to directionally-dependent

477 adsorption on defect surface structures. In both the cases, our model successfully ranks

478 the relative stability of different atomic configurations at a fraction of the computational

479 cost $(\sim 10 \%)$ of exhaustive DFT calculations, thereby providing a framework to identify

480 relevant atomic configurations for surface environments with large and complex

481 configurational spaces. In addition to reducing the overall computational cost, this

482 automated approach reduces the possibility of systematic bias resulting from use of

483 chemical intuition alone to identify structures with target properties. This approach can

484 therefore serve as a starting point for developing detailed atomic description of complex

485 catalyst surfaces under in-situ conditions, help identify interesting regions of the

486 chemical solution space to be investigated with rigorous state-of-the-art methods, 
487 ultimately leading to fundamental insights into factors that govern heterogeneous 488 catalysis in structurally and chemically complex environments. 


\section{Methods}

\section{Dataset}

492 The dataset used for model training and prediction is a collection of a diverse set of 493 calculations corresponding to 1) $\mathrm{NO}^{*}$, varying from 1-6 adsorbates (coverages of 1/12 to $4941 / 2 \mathrm{ML})$, on a $\mathrm{Pt}_{3} \mathrm{Sn}(111)$ surface, and 2) $\mathrm{OH}^{\star}$ surface configurations on $\mathrm{Pt}(100)$ and $495 \mathrm{Pt}(221)$, also encompassing 1-6 adsorbates (coverages of $1 / 12$ to $1 / 2 \mathrm{ML}$ ) - see below for 496 unit cell details). The graph enumeration code, SurfGraph, is used to identify the binding 497 sites and to generate the high coverage configurations which are converted to a graph 498 object through ACE-GCN for property prediction. The target property of choice is the 499 binding energy of the adsorbates, normalized to the number of adsorbates considered 500 in the facet:

\section{DFT methods}

506 The simulations for $\mathrm{NO}^{*}$ on $\mathrm{Pt}_{3} \mathrm{Sn}(111)$ were adopted from previous publications. ${ }^{23}$ For 507 the case of $\mathrm{OH}^{*}$ adsorption on $\mathrm{Pt}(221)$, the simulations are performed within the 508 framework of periodic density functional theory with the Vienna Ab Initio Simulation 509 Package (VASP) . ${ }^{49}$ The energies and geometries of the most stable configurations of $510 \mathrm{OH}^{*}$ on the $\mathrm{Pt}(221)$ surface are obtained through minimization of the total energy with 511 respect to geometry by spin polarized generalized gradient approximation calculations 512 (GGA-PBE). ${ }^{50}$ The projected augmented wave (PAW) method is used to account for the 513 effect of core electrons on the valence electron density. ${ }^{51}$ A PBE-calculated lattice 
514 constant of $3.97 \AA$ for pure Pt is employed. The Pt(221) surface is represented by a $3 \times 3$

515 unit cell with 4 layers (total of 33 atoms per unit cell). A vacuum equivalent to $13 \AA$ is

516 applied between any two successive slabs, and surface relaxation is allowed in the top

517 three layers. A planewave energy cutoff of $300 \mathrm{eV}$ is used for the high-throughput

518 calculations. A minimum k-point grid sampling of $3 \times 3 \times 1$ is employed. For selected cases

519 reported in the phase diagram in Figure 5, a larger unit cell containing $60 \mathrm{Pt}$ atoms is

520 utilized, and a planewave energy cutoff of $400 \mathrm{eV}$, along with k-point grid sampling

$5214 \times 4 \times 1$, is employed. It is observed that between the two different kinds of models and

522 simulation parameters utilized, the trends in the adsorption energies of $\mathrm{OH}^{*}$ remains the

523 same, with minimal $(\sim 0.1 \mathrm{eV})$ change in relative adsorption energies. The electronic

524 occupancies are determined according to a Methfessel- Paxton scheme with an energy

525 smearing of $0.2 \mathrm{eV}$. Dipole corrections are used in all cases to decouple the electrostatic

526 interactions between the periodically repeated slabs. Structures are fully relaxed until

527 the Hellmann- Feynman forces acting on the atoms are smaller than $0.05 \mathrm{eV} / \AA \AA$. Atomic

528 configrations were visualized using Atomic Simulation Environment (ASE) and Ovito. $529 \quad 52,53$

531 Adsorbate subgraph generation

532 Adsorbate subgraphs were generated using the SurfGraph algorithm. ${ }^{41}$ Initially, for a 533 given unit cell, a full graph incorporating all the atoms in the cell is generated. Adsorbate 534 nodes are then identified, and a subgraph is generated with each identified adsorbate 535 node as the center. The subgraphs are generated such that they incorporate the 536 information of the surface atoms immediately adjacent to the adsorbate along with other 537 adsorbate atoms interacting with these surface atoms.

\section{Hydrogen bond generation with directed graphs}

540 All hydrogen atoms with a bond distance greater than $1.3 \AA$ and less than $2.1 \AA$ to a 541 given oxygen atom are constituted as hydrogen bonds. To construct combinations of 542 possible pairs of $\mathrm{H}$-bonds between a set of oxygen atoms, all possible hydrogen bonds 543 are initially identified using the rule explained in the previous sentence. Then, all possible 
544 directed graphs are generated between the identified pairs, using the rule that each $\mathrm{OH}$ 545 adsorbate can only donate one hydrogen bond and accept multiple hydrogen bonds.

546 The directed graph combinations with the maximum number of hydrogen bond pairs are

547 then selected for property prediction or to perform DFT simulations.

\section{Model architecture and implementation}

550 Graph neural networks (GNN), also known as message-passing neural networks, ${ }^{39,54}$ 551 have been previously proposed for computer vision, natural language processing, 552 generating molecular fingerprints, predicting crystal bulk properties, and predicting 553 binding energy on surface slab models. The network developed in this work is the 554 extension of the graph convolution neural network (GCN) approach introduced by Xie et.

555 al. ${ }^{25}$ The GCN framework is coupled with a sub-graph generation routine to 556 systematically encode complex high coverage surface configurations. The subgraphs 557 capture important features of the high coverage geometries, and at the same time, the 558 versatility of the neural networks provides nonlinear mapping between the chemical 559 fingerprints and the target property. Hence, it is possible to strike a balance between 560 end-to-end feature learning, provided by deep neural networks, and chemical intuition 561 found in 'hand-engineered' features.

562 Each crystal lattice entry is split into smaller network motifs (subgraphs) as per 563 the number of unique adsorbates identified by SurfGraph. Each subgraph is an 564 adsorbate-centered undirected graph (ego-graph) with nodes representing the atoms 565 and edges representing the connection between the neighboring atoms in the lattice. 566 The chemical identity of each node in this subgraph is represented by a feature vector 567 generated based on its elemental identity using a combination of chemical and 568 geometric features. These attributes are encoded as one-hot encoding. The edge 569 connecting two nodes is described by edge attributes based on the spatial pairwise 570 atom distance. This feature can be expressed either as a Gaussian feature expansion, 571 as done in previous implementations, ${ }^{25}$ or as one-hot encoding, as implemented in the 572 current version. The reason for using the one-hot encoding expression of the spatial 573 bond distance is to modulate model's sensitivity to bond fluctuations arising out of 
574 structure optimization. A full list of chemical and geometric properties used is provided 575 in the Supplemental Information S2. Next, the bond distance and the one hot encodings 576 are used to create an adjacency matrix for each subgraph. An indexing scheme is 577 generated to account for various neighbors of a given node; each node index is 578 superseded by the adsorbate index based on the number of unique adsorbates in each 579 crystal entry. Likewise, for every node atom and its corresponding neighbors, the atom 580 indices are superseded by a supplemental indexing linking the neighboring atoms to its 581 parent node. This indexing strategy facilitates the subsequent hierarchical pooling 582 operations, enabling the network to account for arbitrary sized subgraphs. A schematic 583 of this pooling operation strategy is provided in the Supplemental Information S2. Model

584 training starts by embedding node attributes in subgraph embeddings. The graph 585 convolution layers iteratively update the node feature vectors by performing convolutions 586 with surrounding nodes in the subgraphs using.

$$
Z_{(i, j)_{k}}^{(t)}=v_{j}^{(t)} \oplus u_{(i, j)_{k}}
$$

592 Equation (1) is the new fingerprint vector formed by concatenation of corresponding 593 neighbor and edge features for each node. Equation (2) shows the graph convolution 594 equation used for iterating the node features in each message-passing round. This 595 equation is inspired from work for predicting small molecule and bulk crystal properties. 596 Here, $W_{x}$ and $b$ are the shared weights and biases for the graph convolution module, 597 while $g_{a c t}$ is the softplus activation function, a smooth approximation of the ReLU 598 (rectified linear unit).

The hierarchical pooling is implemented using PyTorch scatter module's scatter 600 method. Through this method, elements in the input matrix of known dimensions can be 601 reduced (summed or normalized) by explicitly specifying the indices which have been 602 used for the said reduction. As a result, arbitrarily sized subgraphs are collapsed into a 
603 single user-defined n-sized vector fingerprint equivalent to the atom embeddings defined

604 for each atom node at the start. Following the convolution and mean pooling operations, 605 the fingerprint vector is supplied to fully connected layers to capture the mapping of 606 configuration to the target property). The creation of graph objects for the high coverage 607 configurations is parallelized across multiple CPU cores using DASK. ${ }^{55}$

\section{Model training}

610 The network performance is evaluated using three common metrics based on the 611 model's residuals, the mean absolute error (MAE), the root mean-squared error (RMSE), 612 and the mean absolute percentage error (MAPE). A train-validation-test scheme is 613 adopted for choosing the best model for prediction. During the training phase, the data 614 is randomly split into a train-validation-test split where the test set is kept aside for final 615 evaluation. The model weights are iteratively updated by minimizing the loss function 616 (MSE in this case) associated with predicting the target in the training data, and the 617 validation set is scored after each epoch (as per the MAE). The Adam optimizer as 618 implemented in PyTorch is used for the training. After model training for predefined 619 epochs, the model with best validation score is selected for evaluation on the test set. 620 A complete list of hyperparameters is provided in the Supplemental Information S3. 621 Model training and validation was carried on local CPU cores and Tesla P100 GPU cores 622 provided by the Purdue's Research Computing Facility. 


\section{Acknowledgements}

627 The authors acknowledge the United States Department of Energy through the Office of 628 Science, Office of Basic Energy Sciences (BES), Chemical, Biological, and

629 Geosciences Division, Data Science Initiative, grant DE-SC0020381. Use of the Center

630 for Nanoscale Materials, a U.S. Department of Energy, Office of Science, Office of

631 Basic Energy Sciences User Facility under Contract No. DE-AC02-06CH11357, and of

632 computational resources from the National Energy Research Scientific Computing

633 Center, is also acknowledged.

634 
635

636

637

638

639

640

641

642

643

644

645

646

647

648

649

650

651

652

653

654

655

656

657

658

659

660

661

662

663

664

665

666

667

668

669

670

671

672

673

674

675

676

\section{References}

1. Greeley, J. et al. Alloys of platinum and early transition metals as oxygen reduction electrocatalysts. Nature Chemistry 1, 552-556 (2009).

2. Bligaard, T. et al. The Brønsted-Evans-Polanyi relation and the volcano curve in heterogeneous catalysis. Journal of Catalysis 224, 206-217 (2004).

3. Nørskov, J. K. et al. Origin of the Overpotential for Oxygen Reduction at a Fuel-Cell Cathode. The Journal of Physical Chemistry B 108, 17886-17892 (2004).

4. Lansford, J. L., Mironenko, A. V. \& Vlachos, D. G. Scaling relationships and theory for vibrational frequencies of adsorbates on transition metal surfaces. Nature Communications 8, 016105 (2017).

5. Jacobs, R., Hwang, J., Shao-Horn, Y. \& Morgan, D. Assessing Correlations of Perovskite Catalytic Performance with Electronic Structure Descriptors. Chemistry of Materials 31, 785-797 (2019).

6. Back, S. et al. Convolutional Neural Network of Atomic Surface Structures To Predict Binding Energies for High-Throughput Screening of Catalysts. The Journal of Physical Chemistry Letters 10, 4401-4408 (2019).

7. Batchelor, T. A. A. et al. High-Entropy Alloys as a Discovery Platform for Electrocatalysis. Joule (2019) doi:10.1016/j.joule.2018.12.015.

8. Moosavi, S. M. et al. Understanding the diversity of the metal-organic framework ecosystem. Nature Communications 11, 2618-10 (2020).

9. Goodall, R. E. A. \& Lee, A. A. Predicting materials properties without crystal structure: deep representation learning from stoichiometry. Nat Commun 11, 6280 (2020).

10. Saidi, W. A., Shadid, W. \& Castelli, I. E. Machine-learning structural and electronic properties of metal halide perovskites using a hierarchical convolutional neural network. Npj Comput Mater 6, 36 (2020).

11. Back, S., Tran, K. \& Ulissi, Z. W. Discovery of Acid-Stable Oxygen Evolution Catalysts: High-Throughput Computational Screening of Equimolar Bimetallic Oxides. Acs Appl Mater Inter 12, 38256-38265 (2020).

12. Ma, X., Li, Z., Achenie, L. E. K. \& Xin, H. Machine-Learning-Augmented Chemisorption Model for CO2 Electroreduction Catalyst Screening. The Journal of Physical Chemistry Letters 6, 3528-3533 (2015).

13. Lu, Z., Chen, Z. W. \& Singh, C. V. Neural Network-Assisted Development of HighEntropy Alloy Catalysts: Decoupling Ligand and Coordination Effects. Matter 3, 13181333 (2020).

14. Lu, Z., Yadav, S. \& Singh, C. V. Predicting aggregation energy for single atom bimetallic catalysts on clean and $\mathrm{O}^{*}$ adsorbed surfaces through machine learning models. Catal Sci Technol 10, 86-98 (2019).

15. Chowdhury, A. J. et al. Prediction of Adsorption Energies for Chemical Species on Metal Catalyst Surfaces Using Machine Learning. J Phys Chem C 122, 28142-28150 (2018). 
16. Ghanekar, P. et al. Catalysis at Metal/Oxide Interfaces: Density Functional Theory and Microkinetic Modeling of Water Gas Shift at Pt/MgO Boundaries. Topics in Catalysis 63, 673-687 (2020).

680 17. Deshpande, S. \& Greeley, J. First-Principles Analysis of Coverage, Ensemble, and 681 Solvation Effects on Selectivity Trends in NO Electroreduction on Pt 3Sn Alloys. ACS 682 Catalysis 9320-9327 (2020) doi:10.1021/acscatal.0c01380.

683 18. Bruix, A., Margraf, J. T., Andersen, M. \& Reuter, K. First-principles-based multiscale modelling of heterogeneous catalysis. Nature Catalysis 9, 17-12 (2019). 19. Bhandari, S., Rangarajan, S. \& Mavrikakis, M. Combining Computational Modeling with Reaction Kinetics Experiments for Elucidating the In SituNature of the Active Site in Catalysis. Accounts of Chemical Research 53, 1893-1904 (2020). 20. Dionigi, F. et al. In-situ structure and catalytic mechanism of NiFe and $\mathrm{CoFe}$ layered double hydroxides during oxygen evolution. Nature Communications 11, 4347 (2020). 21. Yan, B. et al. Surface Restructuring of Nickel Sulfide Generates Optimally Coordinated Active Sites for Oxygen Reduction Catalysis. Joule 1, 600-612 (2017). 22. Lansford, J. L. \& Vlachos, D. G. Infrared spectroscopy data- and physics-driven machine learning for characterizing surface microstructure of complex materials. Nat Commun 11, 1513 (2020). 23. Deshpande, S., Maxson, T. \& Greeley, J. Graph theory approach to determine configurations of multidentate and high coverage adsorbates for heterogeneous catalysis. npj Computational Materials 6, 79 (2020). 24. Boes, J. R., Mamun, O., Winther, K. \& Bligaard, T. Graph Theory Approach to HighThroughput Surface Adsorption Structure Generation. The Journal of Physical

\section{Chemistry A 123, 2281-2285 (2019).}

25. Xie, T. \& Grossman, J. C. Crystal Graph Convolutional Neural Networks for an Accurate and Interpretable Prediction of Material Properties. Physical Review Letters 120, 1929 (2018).

26. Battaglia, P. W. et al. Relational inductive biases, deep learning, and graph networks. Arxiv cs.LG, (2018).

27. Cybulskis, V. J. et al. Zinc Promotion of Platinum for Catalytic Light Alkane Dehydrogenation: Insights into Geometric and Electronic Effects. ACS Catalysis 7, 4173-4181 (2017).

28. Greeley, J. \& Mavrikakis, M. Alloy catalysts designed from first principles. Nature Materials 3, 810-815 (2004).

29. Purdy, S. C. et al. Origin of Electronic Modification of Platinum in a Pt 3 V Alloy and Its Consequences for Propane Dehydrogenation Catalysis. ACS Applied Energy Materials 3, 1410-1422 (2020). 30. Purdy, S. C. et al. Structural trends in the dehydrogenation selectivity of palladium alloys. Chemical Science 11, 5066-5081 (2020).

31. Clayborne, A., Chun, H.-J., Rankin, R. B. \& Greeley, J. Elucidation of Pathways for NO Electroreduction on Pt(111) from First Principles. Angewandte Chemie 127, 83738376 (2015). 

ultrathin (hydroxy)oxide films on transition metal substrates for electrochemical energy conversion. Nature Energy 2, 17070 (2017). 33. Haid, R. W., Kluge, R. M., Liang, Y. \& Bandarenka, A. S. In Situ Quantification of the Local Electrocatalytic Activity via Electrochemical Scanning Tunneling Microscopy. Small Methods 2000710 (2020) doi:10.1002/smtd.202000710.

34. McCrum, I. T. \& Koper, M. T. M. The role of adsorbed hydroxide in hydrogen evolution reaction kinetics on modified platinum. Nature Energy 39, 163-9 (2020). 35. Wei, J. et al. The Dynamic Nature of CO Adlayers on Pt(111) Electrodes.

Angewandte Chemie 132, 6241-6245 (2020). 36. lovanac, N. C. \& Savoie, B. M. Improving the generative performance of chemical autoencoders through transfer learning. Mach Learn Sci Technology 1, 045010 (2020). 37. Smith, J. S. et al. Approaching coupled cluster accuracy with a general-purpose neural network potential through transfer learning. Nat Commun 10, 2903 (2019). 38. Hutchinson, M. L. et al. Overcoming data scarcity with transfer learning. Arxiv (2017).

39. Gilmer, J., Schoenholz, S. S., Riley, P. F., Vinyals, O. \& Dahl, G. E. Neural Message Passing for Quantum Chemistry. in 1263-1272 (PMLR, 2017).

40. Ahmad, Z., Xie, T., Maheshwari, C., Grossman, J. C. \& Viswanathan, V. Machine Learning Enabled Computational Screening of Inorganic Solid Electrolytes for Suppression of Dendrite Formation in Lithium Metal Anodes. ACS Central Science 4, 996-1006 (2018).

41. Deshpande, S., Maxson, T. \& Greeley, J. Graph theory approach to determine configurations of multidentate and high coverage adsorbates for heterogeneous catalysis. npj Computational Materials 6, 4981 (2020).

42. Zeng, Z. \& Greeley, J. Characterization of oxygenated species at water/Pt(111) interfaces from DFT energetics and XPS simulations. Nano Energy 29, 369-377 (2016). 43. Pfisterer, J. H. K., Liang, Y., Schneider, O. \& Bandarenka, A. S. Direct instrumental identification of catalytically active surface sites. Nature 549, 74-77 (2017).

44. Cheula, R., Soon, A. \& Maestri, M. Prediction of morphological changes of catalyst materials under reaction conditions by combined ab initiothermodynamics and microkinetic modelling. Catal. Sci. Technol. 54, 3465 (2018).

45. Müller, A., Comas-Vives, A. \& Copéret, C. Shape and Surface Morphology of Copper Nanoparticles under CO 2 Hydrogenation Conditions from First Principles. $J$ Phys Chem C 125, 396-409 (2020).

46. Bandarenka, A. S., Hansen, H. A., Rossmeisl, J. \& Stephens, I. E. L. Elucidating the activity of stepped Pt single crystals for oxygen reduction. Physical Chemistry Chemical Physics 16, 13625-13629 (2014).

47. Deshpande, S., Kitchin, J. R. \& Viswanathan, V. Quantifying Uncertainty in Activity Volcano Relationships for Oxygen Reduction Reaction. ACS Catalysis 6, 5251-5259 (2016).

48. Heenen, H. H., Gauthier, J. A., Kristoffersen, H. H., Ludwig, T. \& Chan, K. Solvation at metal/water interfaces: An ab initiomolecular dynamics benchmark of common computational approaches. The Journal of Chemical Physics 152, 144703 (2020). 
763 49. Kresse, G. \& Furthmüller, J. Efficient iterative schemes for ab initiototal-energy

764 calculations using a plane-wave basis set. Physical Review B 54, 11169-11186 (1996).

765 50. Perdew, J. P., Burke, K. \& Ernzerhof, M. Generalized Gradient Approximation Made

766 Simple. Physical Review Letters 77, 3865-3868 (1996).

767 51. Kresse, G. \& Joubert, D. From ultrasoft pseudopotentials to the projector

768 augmented-wave method. Physical Review B 59, 1758-1775 (1999).

769 52. Larsen, A. H. et al. The atomic simulation environment - a Python library for

770 working with atoms. Journal of Physics: Condensed Matter 29, 273002 (2017).

771 53. Stukowski, A. Visualization and analysis of atomistic simulation data with OVITO-

772 the Open Visualization Tool. Model Simul Mater Sc 18, 015012 (2010).

773 54. Río, E. G. del, Mortensen, J. J. \& Jacobsen, K. W. Local Bayesian optimizer for

774 atomic structures. Physical Review B 100, 104103 (2019).

775 55. Team, D. D. Dask: Library for dynamic task scheduling. https://dask.org (2016). 